\title{
Priming and property dominance effects in semantic memory
}

\author{
MARK H. ASHCRAFT \\ Cleveland State University, Cleveland, Ohio 44115
}

\begin{abstract}
Retrieval from semantic memory was examined by means of reaction times to property statements (e.g., sparrow has beak). The variables of interest were normatively defined property dominance (frequency), type of priming between related sentences (sparrow-sparrow vs. sparrow-robin), and separation or lag between related sentences. Statements asserting a high-dominant property (1) were verified more quickly than those containing low-dominant properties, (2) were primed by a preceding related sentence of either high or low dominance, and (3) revealed decay of priming from Lag 1 to 4 . The differences between priming with and without stimulus repetition were nonsignificant when stimuli were treated as a random effect. In support of the Collins and Quillian (1972) model of semantic memory, the priming and property dominance factors interacted.
\end{abstract}

The present studies involve an investigation of priming and property dominance effects in the verification of property statements. Priming is understood to be a process by which concepts and their meanings in semantic memory are activated, regardless of the origin of that activation (Collins \& Quillian, 1972). For example, the concept BIRD might be primed by the words bird, robin, or wings, by seeing an actual bird, etc. Priming is generally examined in a Stimulus 1 /Stimulus 2 paradigm, in which performance on the second stimulus is altered as a function of the information provided by the first stimuius. A significant decrease in reaction time (RT) to the second stimulus, for example, constitutes the typical evidence for priming (e.g., Rosch, 1975). Property dominance here refers to the proposition that some properties or attributes of concepts dominate the characterization of those concepts, in that they are more important, frequent, necessary, or otherwise salient with respect to those concepts. For example, wings is a more dominant or frequently stated property of bird than is mouth, although both are true properties of the concept. In a statement verification task, Conrad (1972) and Glass, Holyoak, and O'Dell (1974) have shown that sentences containing properties of high production frequency (high property dominance) can be verified more rapidly than those with low-frequency

This research was partially supported by U.S. Public Health Service Grants HD-00183 and HD-00870 from the National Institute of Child Health and Human Development to the Bureau of Child Research, University of Kansas. It formed the basis of a dissertation submitted to the University of Kansas in partial fulfillment of the requirements for the PhD. I would like to thank my committee chairman, George Kellas, and members, James Juola, Edwin Martin, Edward Wike, and Margaret Schadler, for their help and advice on this project. Reprint requests should be addressed to the author, Department of Psychology, Cleveland State University, East 24th \& Euclid, Cleveland, Ohio 44115. properties. This effect suggests that property dominance can be considered analogous to the more general variable of semantic similarity or relatedness (Kintsch, 1974 Rips, Shoben, \& Smith, 1973).

In the context of the Collins and Quillian (1972 model of semantic memory, priming and property dom. inance effects may be considered central to the issue: of process and structure, respectively. Collins and Quillian (1972; see also Collins \& Loftus, 1975) have proposed a model in which the spread of activation ot priming among concept nodes represents the basic mechanism for accessing knowledge. In a network structure organized along the dimension of semantic relatedness, the meaning of a concept may be repre. sented as that constellation of other concepts (or "prop. erties" generically) to which it is related, along with the particular types of relations involved (i.e., superset, part of). Given these sets of concept-relation-property links, semantic relatedness can be structurally represented as the accessiblity of the relational path or, conversely, as distance between concept and property. Operationally, semantic relatedness is reflected by the property dominance or frequency value, while semantic distance reflects the inverse of dominance measures. Given this structure, Collins and Quillian proposed that the spread of activation or priming represents the basic mechanism of accessing semantic information. Activation spreads down the relational paths, proportional to the accessibility or distance to be traversed, resulting in greater priming of properties which are highly related (dominant) to the concept. This priming should then affect the processing of a subsequent stimulus which involves an already primed property or concept, and the priming effect should be proportional to the degree of activation. Accordingly, the Collins and Quillian (1972) model predicts an interaction between priming and dominance-priming effects should be maximal under conditions of high semantic relatedness, but only mod- 
derate (or possibly negative; Loftus, 1975) under lowrelatedness conditions.

The primary theoretical rival of the Collins and Quillian (1972) model is the Smith, Shoben, and Rips (1974) feature comparison model. In this model, a concept is represented by a list of semantic features or attributes, rather than as a conceptual node in a network. According to the model, verifying a relationship between two concepts (i.e., "A robin is a bird") involves feature set comparisons which indicate the amount of overlap or semantic similarity between the two concepts. While the model originally emphasized the verification of superordinate statements, a recent revision (Smith, Rips, \& Shoben, 1974) extended the model to encompass the verification of property statements. Although the revision involves many additions to the basic feature comparison model, it does account for the effects of semantic relatedness on property statement verification; i.e., a statement involving a highly related concept and property should be verified more quickly than one asserting a low-related concept and property. Despite this revision. however, no obvious scheme emerges from the model to account for semantic priming or its possible interdependence with semantic related. ness. That is, Smith et al. have apparently limited their concern to an explanation of phenomena occurring within a single trial, to the exclusion of effects such as priming, which occur across trials. Thus, further revisions, or at least more detailed specification, of the Smith et al. model will be necessary to the degree that significant priming effects, and any interrelationships between priming and property dominance, can be demonstrated in a sentence verification task.

Despite the theoretical interdependence of priming and property dominance in Collins and Quillian (1972), experimental investigations have not generally considered these variables jointly. As noted. Conrad (1972) and Glass et al. (1974) have reported a property dominance effect on RT in a verification task: that is, when subjects were asked to verify the truth of a statement like " $A$ robin has wings," RT increased as the asserted property decreased in normative frequency or dominance. Semantic priming was not manipulated in either of these reports, however. Conversely, Collins and Quillian (1970) presented pairs of related sentences ("A canary is a bird"-"A canary has wings") in order to investigate verification processes in the presence of priming due to semantic relatedness. Their results revealed a marked decrease in Sentence $2 \mathrm{RT}$-in other words, priming of Sentence 2 as a function of the semantically related Sentence 1 . Unfortunately, property dominance/frequency was an uncontrolled factor in this experiment (see Conrad, 1972), thereby undermining the generality of the results to property verification and priming processes. Further, the procedure of repeating part of Stimulus 1 in the second sentence ("canary"), for all pairs of related sentences, intro- duced the possibility that at least part of the observed priming effect might be due instead to repetition alone (see Forbach, Stanners, \& Hochhaus, 1974).

It is the contention of the present paper that priming and property dominance variables must be evaluated jointly, given their theoretical interdependence in Collins and Quillian (1972). Such an evaluation must first be based on a normatively defined, rather than intuitively generated, sample of stimuli in which property dominance can be controlled. The normative and validation experiments to be presented were designed to satisfy this criterion. Following this, two experiments are reported in which the joint effects of priming and property dominance are examined as a function of the type of semantic relationship involved-that is, related pairs of sentences with and without stimulus repetitionand the decay interval or lag between the members of related sentence pairs.

\section{NORMATIVE AND VALIDATION EXPERIMENTS}

In order to provide an objective source of property statements, normative data on properties were collected. Briefly, 35 undergraduates were given booklets with a concept name at the top of each page. A total of 140 concept names were used, 20 superordinates and six exemplars of each superordinate category. The six exemplars were divided into three groups, two highfrequency category members, two medium, and two low (Battig \& Montague, 1969). For each category, a subject listed the properties of either the superordinate or its six exemplars. Thus, each subject listed properties for 70 concepts, 10 superordinates and the 60 exemplars from the remaining categories. Subjects were given $1 \mathrm{~min} /$ page in which to list all the properties they could think of. Instructions were provided to insure that properties, rather than exemplars or word associations, would be given. The instructions also emphasized that subjects should list a property even though they might have already listed it for a different word.

Responses were tabulated in order to determine the frequency of occurrence of each property in relation to its concept. With few exceptions, the property dominance hierarchy for each concept included from one to five properties listed by at least $50 \%$ of the normative sample. Many other properties were less common, being listed by a minority of the subjects. Of incidental interest is the fact that $70 \%$ of the rated concepts elicited the concept's superordinate as one of the highdominant properties (i.e., properties listed by $50 \%$ or more of the subject sample). This finding is in agreement with Conrad's (1972) report of superordinate normative responses, and reaffirms the possible utility of considering the superordinate simply as a high-frequency property of a concept (but cf. Smith, Shoben. \& Rips. 1974). Table 1 lists the concepts and properties, and the normative frequency of occurrence, for the pool of 
Table 1

Normative Frequency of Occurrences (Expressed as a Percentage) for Properties in Relation to Concepts

\begin{tabular}{|c|c|c|c|c|c|c|c|c|c|c|c|}
\hline \multirow[b]{2}{*}{ Concept } & & \\
\hline & Property & $\begin{array}{l}\text { Per- } \\
\text { cent }\end{array}$ & Concept & Property & $\begin{array}{l}\text { Per- } \\
\text { cent }\end{array}$ & spoon & $\begin{array}{l}\text { metal } \\
\text { rounded }\end{array}$ & $\begin{array}{l}75 \\
25\end{array}$ & nail & $\begin{array}{l}\text { head } \\
\text { point }\end{array}$ & $\begin{array}{l}62 \\
54\end{array}$ \\
\hline \multirow[t]{6}{*}{ oak } & leaves & 69 & \multirow[t]{6}{*}{ carrot } & orange & 87 & & $\begin{array}{l}\text { smooth } \\
\text { shiny }\end{array}$ & $\begin{array}{r}17 \\
8\end{array}$ & & $\begin{array}{l}\text { bend } \\
\text { rust }\end{array}$ & $\begin{array}{l}8 \\
8\end{array}$ \\
\hline & branches & 50 & & long & 56 & & curved & 8 & & & \\
\hline & brown & 19 & & underground & 25 & \multirow{4}{*}{ gun } & & 50 & & & \\
\hline & lumber & 12 & & crisp & 18 & & trigger & 59 & & & \\
\hline & limbs & 6 & & crunchy & 12 & & & $\begin{array}{l}50 \\
17\end{array}$ & & & \\
\hline & & & & vitamins & 6 & & $\begin{array}{l}\text { steel } \\
\text { noise }\end{array}$ & $\begin{array}{r}17 \\
8\end{array}$ & & & \\
\hline \multirow[t]{4}{*}{ maple } & wood & 50 & \multirow[t]{4}{*}{ potato } & starch & 62 & \multirow{4}{*}{ knife } & holster & 8 & & & \\
\hline & grow & 12 & & brown & $\begin{array}{l}56 \\
25\end{array}$ & & sharp & 100 & & & \\
\hline & tall & 12 & & skin & $\begin{array}{l}25 \\
18\end{array}$ & & blade & 54 & & & \\
\hline & shade & & & $\begin{array}{l}\text { cooked } \\
\text { edible }\end{array}$ & $\begin{array}{l}18 \\
12\end{array}$ & & shiny & 25 & & & \\
\hline \multirow[t]{2}{*}{ rose } & petals & 67 & \multirow[t]{2}{*}{ milk } & white & 100 & & $\begin{array}{l}\text { nard } \\
\text { dangerous }\end{array}$ & 8 & & & \\
\hline & buds & 11 & & liquid & 53 & & & & & & \\
\hline
\end{tabular}

$\begin{array}{llrlll} & \text { pollen } & 11 & & \text { healthy } & 18 \\ \text { daisy } & \text { stem } & 67 & \text { Coke } & \text { drink } & 50 \\ & \text { smell } & 22 & & \text { sugar } & 21 \\ & \text { roots } & 17 & & \text { carbonated } & 21 \\ & \text { wilt } & 6 & & \text { cold. } & 14 \\ & & & & \text { syrup } & 14 \\ \text { apple } & \text { red } & 92 & \text { airplane } & \text { wings } & 78 \\ & \text { seeds } & 54 & & \text { engines } & 50 \\ & \text { juicy } & 23 & & \text { seats } & 50 \\ & \text { core } & 23 & & \text { fast } & 21 \\ & & & & \text { fuel } & 21 \\ \text { pear } & \text { yellow } & 77 & \text { car } & \text { wheels } & 67 \\ & \text { sweet } & 23 & & \text { seat } & 60 \\ & \text { soft } & 15 & & \text { windows } & 20 \\ & \text { peel } & 8 & & \text { brakes } & 20 \\ \text { horse } & \text { tail } & 67 & \text { trout } & \text { scales } & 58 \\ & \text { hooves } & 60 & & \text { tail } & 25 \\ & \text { nose } & 20 & & \text { bones } & 8 \\ & \text { eat } & 7 & & \text { slimy } & 8\end{array}$

$\begin{array}{llllll}\operatorname{dog} & \text { ears } & 50 & \text { bass } & \text { fins } & 55 \\ \text { claws } & 13 & & \text { gills } & 25 \\ & \text { bite } & 13 & & \text { swim } & 11\end{array}$

$\begin{array}{llrllr}\text { sparrow } & \text { beak } & 65 & \text { ant } & \text { small } & 65 \\ & \text { small } & 65 & & \text { black } & 55 \\ & \text { chirp } & 23 & & \text { body } & 25 \\ & \text { eggs } & 23 & & \text { crawls } & 10 \\ \text { robin } & \text { fly } & 76 & \text { bee } & \text { sting } & 95 \\ & \text { eyes } & 12 & & \text { honey } & 58 \\ & \text { feet } & 6 & & \text { worker } & 16 \\ & \text { colorful } & 6 & & \text { fuzz } & 10 \\ & & & & \text { head } & 5 \\ \text { house } & \text { floor } & 50 & \text { chair } & \text { legs } & 81 \\ & \text { walls } & 25 & & \text { arms } & 25 \\ & \text { yard } & 8 & & \text { cushion } & 12 \\ & \text { attic } & 8 & & \text { moveable } & 6 \\ \text { apartment } & \text { bedroom } & 67 & \text { sofa } & \text { soft } & 69 \\ & \text { kitchen } & 58 & & \text { cloth } & 25 \\ & \text { rooms } & 17 & & \text { large } & 19 \\ & \text { furnished } & 17 & & \text { material } & 19 \\ & \text { door } & 8 & & & \\ & \text { prongs } & 80 & \text { hammer } & \text { handle } & 54 \\ \text { fork } & \text { handle } & 50 & & \text { hard } & 54 \\ & \text { silver } & 10 & & \text { heavy } & 23 \\ & \text { light } & 10 & & \text { useful } & 23 \\ & & & & \text { helpful } & 8 \\ & & & & & \end{array}$

Table 1

Continued

concept-property pairs used in the subsequent experiments. Notice that this table presents only a portion of the responses to any one concept noun, and that only concept nouns of high frequency in their respective categories (Battig \& Montague, 1969) were used.

A validation experiment was performed to assess the psychological importance of normatively defined property dominance. The results of this study were expected to demonstrate a significant effect of property dominance on the verification of property statements. The methodology is described here in some detail since the subsequent experiments used highly similar procedures.

\section{Method}

Subjects. Eight undergraduates, enrolled in the introductory class credit.

Stimuli. The two high-frequency category members from 15 of the rated categories were selected from the normative sample (the remaining five categories-minerals, metals, precious stones, fuels, and snakes-were eliminated due to insufficient normative responses). For each concept noun, two properties were chosen from the property dominance hierarchy. One of the two was generated by $50 \%$ or more of the normative sample and served as a high-dominant property for that noun. The second property was generated by $25 \%$ or less of the normative sample and was designated a low-dominant property for the noun. Thus, 60 stimuli formed the pool of true noun-property pairs. A t test revealed no reliable relationship between word frequency (Kučera \& Francis, 1967) and property dominance, $t<1.0$

False stimuli were constructed by re-pairing ail 30 nouns and 60 properties. Therefore, the entire stimulus pool contained 120 noun-property pairs, half of which were true. Half of the pairs contained a high-dominant property, and half a low-dominant property, although in false pairs the property was dominant to an unstated noun (i.e., the two stimulus pairs with "beak" were high dominant, although the dominance of "beak" was defined solely in terms of "sparrow"). Each subject noun appeared four times across the experiment-in a true-high dominant, a true-low dominant, a false-high dominant, and a falselow dominant pair. Each property appeared two times, once in a true pairing and once in a false pairing.

The stimulus sequence consisted of a random ordering of the 120 pairs, with the following restrictions: (1) true-high and true-low pairs with the same subject noun appeared in different halves of the 120 stimuli (Set 1 vs. Set 2); (2) within each set, each noun and property appeared twice; (3) at least four psychology course at the University of Kansas, participated for intervening trials separated nouns from the same category 
(e.g., "robin" and "sparrow"); and (4) at least four trials intervened between pairs containing the same words. Accordingly, there were two sets of 60 pairs, each set containing an equal number (15) of true-high, true-low, false-high, and false-low pairs.

Finally, in addition to the subject noun and property, each stimulus included the appropriate connective verb for the stated property; e.g., "robin has wings," "gun is steel," "dog has wheels," and "trout can fly." This procedure eliminated the necessity to instruct subjects in the generation of appropriate connectives (cf. Kintsch, 1974, Chapter 10). The three-word "telegraphic sentences" were typed in lowercase letters onto transparency paper and fitted into $35-\mathrm{mm}$ slide frames. The three words were typed in a column, with the noun on top, connective just below, and property on the bottom.

Apparatus. Stimuli were rear-projected onto a screen approximately $75 \mathrm{~cm}$ from the subject. The subjects depressed a white button with their nondominant hand, initiating a 2-sec blank interval, followed by presentation of the stimulus. A photoelectric cell triggered the electronic timer as the stimulus appeared, and depression of either telegraph key stopped the timer. The two telegraph keys, with $10 \mathrm{~cm}$ between centers, were located directly in front of the subject and were labeled "true" and "false." Half of the subjects responded to the right key for true sentences, and half to the left. Subjects responded with their dominant hand, which was placed directly in front of the two telegraph keys. Reaction times were recorded to the nearest millisecond.

Design and procedure. The design included one betweensubjects variable, Set Order (Set 1 first vs. Set 2 first), and three within-subjects variables, Set ( 1 vs. 2 ), True/False judgments, and Property Dominance (high vs. low). For stimuli (Clark, 1973), sentences were nested within the Set by True/False by Property Dominance matrix, while the Set Order factor was a within-stimuli factor.

Subjects were tested individually in a darkened room. They were told they would see a series of "telegraphic sentences" (example provided), and that their task was to decide whether the sentence was generally true or false. Subjects were asked to disregard unusual or metaphorical meanings of the words. Speed and accuracy were emphasized equally. Fifty practice trials preceded the 120 experimental sentences. The first 20 practice stimuli were the words "True" and "False;" the remaining practice stimuli were three-word sentences constructed in an identical fashion to the actual test items, but using a different set of concept-property pairs. A brief rest period followed the practice set, and a similar rest intervened between the experimental sets. A signal from the experimenter indicated that the subject should press the white button to initiate a trial. Feedback concerning errors was given immediately throughout the session. Sessions lasted approximately $30 \mathrm{~min}$.

\section{Results and Discussion}

The overall error rate was $2.2 \%$; experimenter and equipment errors resulted in the loss of nine additional scores, bringing the percentage of missing observations up to $3.1 \%$. Given this low percentage of errors, no analysis of error proportions was performed.

Since the error rate was minimal, the unaveraged data were analyzed in an analysis of variance which simultaneously evaluated both Subjects and Stimuli as random factors. Missing scores were replaced with the stimulus mean for that cell, and appropriate degrees of freedom were subtracted. The resultant mean squares were combined into $F^{\prime \prime}$ ratios (Winer, 1971, p. 377) in order to generalize treatment effects simultaneously to new subject and stimulus samples. As a check on the substitution procedure for missing scores, the minimum $F^{\prime}$ ratio was also calculated, as suggested by Clark (1973). The $\min F^{\prime}$ requires two sets of analyses, one which considers Subjects as the random factor (the F1 analysis) and one with Stimuli as the random factor (F2). Since the min $F^{\prime}$ and $F^{\prime \prime}$ procedures yielded redundant patterns of significance, only the $\mathrm{F}^{\prime \prime}$ ratio will be presented as evidence of simultaneous generality across subjects and stimuli. ${ }^{1}$ Significant effects in the F1 and F2 analyses will also be reported when different than the $F^{\prime \prime}$ results.

The main effects of Set and Set Order, as well as their interaction, were highly significant in the $F 2$ analysis; for the interaction, $F 2(1,112)=8.79, p<.005$, $\mathrm{MS}_{\mathrm{e}}=27,067.9$. Inspection of the means suggested that responses to the second presented set were faster than those to the first presented set, regardless of which set came first. This effect was not significant in the F1 analysis, and was also nonsignificant with the $\mathrm{F}^{\prime \prime}$ statistic $\left(\mathrm{F}^{\prime \prime}<1.0\right)$.

In general, high-dominant property statements were verified more quickly than low-dominant statements. This effect was qualified by the Property Dominance by True/False interaction $\left[F^{\prime \prime}(1,73)=4.48, p<.05\right.$, $\mathrm{MS}_{\mathrm{T}}=1,129,155.9, \quad \mathrm{MS}_{\mathrm{e}(\mathrm{s})}=60,540.3, \quad \mathrm{MS}_{\mathrm{e}(\mathrm{st})}=$ $212,180.6]$, displayed in Table 2. The interaction suggested that normative property dominance affected performance only on true sentences, with high-dominant true statements showing a 123-msec advantage in verification time over low-dominant true sentences. False judgments apparently were not influenced by the dominance factor, and in general were judged only somewhat faster $(33 \mathrm{msec})$ than low-dominant true sentences. Finally, the Set by Property Dominance interaction $\left[\mathrm{F}^{\prime \prime}(1 ; 37)=3.22, \mathrm{p}<.10, \mathrm{MS}_{\mathrm{T}}=1,005,956.0\right.$ $\left.\mathrm{MS}_{\mathrm{e}(\mathrm{s})}=128,641.3, \quad \mathrm{MS}_{\mathrm{e}(\mathrm{st})}=212,180.6\right]$ indicated that the dominance effect was somewhat attenuated in Set 2, although the same increasing pattern of RT for true judgments was obtained.

The primary goal of these studies was to establish and validate a normative property dominance hierarchy. The results suggested that the normative data do reflect a valid frequency-to-semantic dominance relationship which characterizes information accessibility in semantic memory. While the results do not discriminate among

Table 2

Means (in Milliseconds), Standard Deviations, and Error Rates by Treatment Groups for the Validation Experiment

\begin{tabular}{lccrrr}
\hline & \multicolumn{2}{c}{ True } & & \multicolumn{2}{c}{ False } \\
\cline { 2 - 3 } \cline { 5 - 6 } & HD & LD & & HD & LD \\
\hline Mean & 1371 & 1494 & & 1477 & 1460 \\
SD & 300 & 360 & & 290 & 291 \\
Error Percent & 2.5 & 3.3 & & 1.7 & 1.3 \\
\hline
\end{tabular}

Note $-H D=$ high dominant, $L D=$ low dominant . 
current models of semantic memory, they do provide the basis for a more analytic evaluation of those models. The following experiments attempted to provide such an evaluation.

\section{EXPERIMENT I}

One difficulty with the Collins and Quillian (1970) demonstration of priming involved repetition of part of the first stimulus in the second target stimulus. As noted, the subject noun in the stimulus sentences was always repeated in the second related stimulus, thereby technically confounding semantic relatedness with repetition in that report. Similar manipulations of priming/repetition are found in Haviland and Clark (1974) and Loftus and Loftus (1974). The point here is simply that such procedures may have biased the estimates of priming effects per se and, in any event, do not provide clear evidence of the importance of semantic priming.

At a more theoretical level, however, pairs of related sentences which include repetition might be more adequately described as maximal or "repeated" priming pairs. In other words, if priming can be considered a continuous effect, stimulus repetition would provide additional semantic priming superimposed on priming due to relatedness. The idea here is that if a concept can be primed by a related concept, then repeating the concept would represent maximum possible priming of that concept. Note, however, that such a procedure may yield misleading estimates of the magnitude of priming effects and characteristics of the decay of priming. Further, such repetition is not a necessary aspect of priming within the Collins and Quillian model: Any path in the network can presumably carry activation to other concepts. With few exceptions (e.g., Meyer \& Schvaneveldt, 1971; Rosch, 1975), though, semantic priming in a Stimulus $1 /$ Stimulus 2 paradigm has apparently not been studied without "repeated priming" or repetition. For both methodological and theoretical reasons, then, there clearly needs to be a manipulation of relatedness between stimuli which is independent of repetition/repeated priming.

The first major experiment to be reported here examined semantic priming between related stimuli in the absence of any stimulus repetition (e.g., "maple is wood"-."oak has leaves"). This procedure provides a more stringent test of the importance of semantic priming than the situation that includes stimulus repetition. In order to examine the predicted interdependence of priming and semantic relatedness, the property dominance factor, established in the validation experiment, was manipulated at both the Sentence 1 and the Sentence 2 positions. This design permitted an evaluation of any differential priming from high- and lowdominant sentences as well as to high- and low-dominant sentences.
Given the results of the validation study, it was predicted that low-dominant pioperty statements would be verified more slowly than high-dominant statements (see also Conrad, 1972; Glass et al., 1974). More interestingly, priming effects were expected to differ as a function of the dominance of the preceding sentence. The general processing assumption (Collins \& Quillian, 1972 ) is that more priming should spread down a highly related, short path, and will consequently persist for a longer interval at those concepts which are closely related to the first stimulus. Accordingly, a highdominant second sentence which follows a highdominant first sentence should be the fastest critical sentence to be verified; that is, the concepts stated in Sentence 2 are more likely to receive a high degree of activation from Sentence 1. For example, "Robin has wings," as a high-dominant Sentence 1, should prime BIRD and FEATHERS to a large extent, and these may in turn prime SPARROW and TAIL, among others; when followed by "Sparrow has feathers," as a highdominant Sentence 2, verification RT should be greatly facilitated due to the already activated SPARROW and FEATHERS concepts. With the same first sentence, followed by "Sparrow has eyes" (a low-dominant Sentence 2), however, relatively little priming should have spread to EYES from ROBIN, although SPARROW may still receive activation. This pattern of activation should result in a reduced priming effect for low-dominant second sentences.

Predictions for the conditions in which low-dominant sentences appear first are less obvious. While it is clear that a low-dominant/low-dominant pair should result in the slowest verification time, the degree of priming from a low-dominant first sentence to a high-dominant second sentence cannot be determined a priori. That is, a low-dominant prime may activate a high-dominant target (particularly the subject noun) to some extent, resulting in priming facilitation; or the high-dominant target may be accessible enough that any priming from the first sentence may be inconsequential. In other words, a prediction of an observable priming effect, over and above the predicted property dominance effect, cannot be made with any certainty for the low-to highdominant priming condition.

Alternate, and less ambitious, predictions from the Collins and Quillian (1972) model may be equally valid in this situation, however. While priming should spread from both the concept and the property nodes, it might be maintained that priming from the property should spread more diversely; in other words, activation from WINGS will spread not only to various bird concepts, but also to insects, airplanes, etc. This situation is analogous to the notion of "fanning" (Anderson, 1974), in which a predicate concept is linked to several subject concepts. Such fanning or diverse spread of activation would result in greater functional importance of the priming released by the subject concept. If such a 
process occurs, then no special advantage for high vs. low first sentences might be expected, assuming comparable degrees of fanning. In this case, priming and property dominance effects should influence performance independently, whereas the first set of predictions implies an interactive relationship as well.

\section{Method}

Subjects. Sixteen undergraduates, enrolled in the introductory psychology course at the University of Kansas, participated for class credit.

Stimuli. New stimulus sentences, constructed in an identical fashion to that described earlier, were added to the pool of stim. uli from the validation experiment. In order to control for the possibility that priming might spread from the property as well as from the subject noun, properties listed under the appropriate superordinate, as well as under the subject noun concept itself were used whenever possible. Eighty-six percent of the sentences conformed to this restriction; the remaining cases reflected subject noun concepts for which no twice-listed property of either dominance level was available. As before, property dominance and word frequency (Kučera \& Francis, 1967) were not statistically related $(t<1.0)$.

A total of 30 stimuli served as critical second sentences which followed 30 related first sentences directly; all 60 sentences were true. An additional 30 true sentences were immediately followed by 30 related but false sentences ("distractor pairs"). As such, true/false judgements were not predictable by semantic relatedness between sentence pairs. An example of a critical pairing is "maple is wood"-"oak has leaves," and a distractor pairing "oak has branches"-"maple has wings." Finally, 60 false sentences, again involving repairing of the subject nouns and properties, were added to equalize the number of true and false trials. Each subject noun appeared in three true and three false sentences, and each property appeared twice.

Since the validation study, and other pilot data, indicated the need for multiple observations per treatment cell, Sentence 1 dominance was manipulated between subjects (and between stimuli). The 30 critical Sentence 2 stimuli (15 high and 15 low dominant) were the same for all subjects (within subjects), although Sentence 2 dominance was, of course, a betweenstimuli factor. Given the limitations on the number of true stimuli that could be constructed, the two sets of first sentences (i.e., those preceding critical stimuli and those preceding false related distractors) were interchanged for the two levels of Sentence 1 dominance. That is, under the high-dominant Sentence 1 condition, the 30 true sentences which preceded the false distractors were of low property dominance. These Sentence 1 sets were interchanged in order to construct the lowdominant Sentence 1 stimulus sequences.

Fifteen randomly selected true sentences from each level of property dominance served as the critical stimuli. These were immediately preceded by related true sentences of either high or low dominance. Distractor pairs of sentences were formed in the same fashion. These critical and distractor pairs, as well as the 60 additional false sentences, were randomly ordered with the following restrictions: No more than four true or four false trials appeared consecutively and, with the exception of critical and distractor pairs, at least eight trials intervened between related nouns. The sequence of 180 trials was divided into fourths for ease of presentation.

Apparatus. Two changes were made to the previously described apparatus. First, since it was necessary to control the intertrial interval in the presence of the priming manipulation, a relay rack was programmed to present a new stimulus 5 sec after the subject's response to the previous sentence. The experimenter recorded the subjects' RT and accuracy during this interval. Second, the telegraph keys were replaced with micro- switch keys, and subjects used both index fingers for responding. As before, half of the subjects responded "true" to the right key and half to the left key.

Design and procedure. Since the validation and pilot research failed to reveal significant treatment effects on false sentences, only RT to true sentences was of interest here. Two independent variables, in addition to Sentence 1 vs. Sentence 2 position, were manipulated: property dominance of Sentence 1 (high vs. low) was a between-subjects between-stimuli factor, and property dominance of Sentence 2 was a within-subjects between-stimuli factor.

Subjects were tested individually, and the instructions and practice stimuli from the validation study were used. Each subject received one of two different orderings of the four sets of stimuli. Due to the 5-sec pacing of the trials, feedback concerning errors was withheld until the end of each set; in most cases, however, the feedback was redundant with the subject's own memory of this performance. Sessions lasted approximately $45 \mathrm{~min}$.

\section{Results and Discussion}

Overall error rate was $2.7 \%$; error rate on critical stimuli was $2.1 \%$. Equipment and experimenter errors brought the percentage of missing scores to $2.8 \%$. Inspection of error proportions by treatment cells revealed nearly equivalent error rates for each condition. Consequently, no analysis of error rate was performed.

The analysis of primary interest compared RT to true first and second sentences as a function of the dominance of these two sentences. Since the same highand low-dominant stimuli served as critical sentences, but different stimuli served as their primers in the two Sentence 1 dominance groups, an overall $\mathrm{F}^{\prime \prime}$ analysis would not be appropriate. That is, the observations at the Sentence 1 position were between stimuli with respect to dominance and, hence, with respect to subjects, while the Sentence 2 observations were between stimuli but within subjects with respect to property dominance. Separate $F^{\prime \prime}$ analyses for the first and second sentence data, however, will be reported. Additionally, an $\mathrm{F}^{\prime \prime}$ analysis was appropriate to the half of the data in which property dominance did not change from Sentence 1 to 2 . This analysis provided an $F^{\prime \prime}$ value for the Sentence 1 vs. 2 factor when dominance was either high or low for both sentence positions. As before, stimulus cell means were substituted for missing scores and appropriate degrees of freedom were subtracted.

In the major analysis, Sentence 2 RT was significantly faster than Sentence 1 RT $[F 1(1,14)=55.75$, $\left.\mathrm{p}<.001, \mathrm{MS}_{\mathrm{e}}=886.9\right]$, thereby confirming that a highly reliable priming effect can be demonstrated without the involvement of stimulus repetition. Second, low-dominant sentences were verified more slowly than high-dominant sentences $[F 1(1,14)=15.86, p<.005$, $\left.\mathrm{MS}_{\mathrm{e}}=2,126.1\right]$. Finally, the interaction of Sentence Position by Property Dominance was significant $\left[\mathrm{F} 1(1,14)=11.48, \mathrm{p}<.005, \quad \mathrm{MS}_{\mathrm{e}}=1,956.0\right]$. This interaction revealed a $93-\mathrm{msec}$ decrease from Sentence 1 to 2 when Sentence 2 asserted a high-dominant property, but only an 18-msec decrease when Sentence 2 stated a low-dominant property. The reliability of this 
interaction was also revealed in the $\mathrm{F}^{\prime \prime}$ analysis conducted on those conditions in which property dominance did not change across sentences; the decrease in RT from Sentence 1 to $2\left[F^{\prime \prime}(1,70)=2.86, p<.10\right.$, $\mathrm{MS}_{\mathrm{T}}=381,940.8, \mathrm{MS}_{\mathrm{e}(\mathrm{s})}=25,262.6, \mathrm{MS}_{\mathrm{e}(\mathrm{st})}=$ $123,548.3]$ was particularly strong for the highdominant sentence portion of the data $\left[\mathrm{F}^{\prime \prime}(1,34)=3.80\right.$, $\mathrm{p}<.06, \mathrm{MS}_{\mathrm{T}}=528,563.2, \mathrm{MS}_{\mathrm{e}(\mathrm{s})}=20,506.8, \mathrm{MS}_{\mathrm{e}(\mathrm{st})}=$ $127,989.4]$, but was nonsignificant for the low-dominant sentence condition, $\mathrm{F}^{\prime \prime}<1.0$.

Property dominance effects were significant in the two $F^{\prime \prime}$ analyses which considered the two sentence positions separately $\left[\mathrm{F}^{\prime \prime}(1,52)=6.20, \mathrm{p}<.025, \mathrm{MS}_{\mathrm{T}}=\right.$ $2,494,128.4, \mathrm{MS}_{\mathrm{e}(\mathrm{s})}=119,750.3, \mathrm{MS}_{\mathrm{e}(\mathrm{st})}=291,506.6$, for all first sentences, and $F^{\prime \prime}(1,35)=4.54, p<.05$, $\mathrm{MS}_{\mathrm{T}}=929,192.0, \quad \mathrm{MS}_{\mathrm{e}(\mathrm{s})}=25,520.3, \quad \mathrm{MS}_{\mathrm{e}(\mathrm{st})}=$ $187,640.6$, for critical second sentences]. In the Sentence 1 analysis, the dominance effect was $125 \mathrm{msec}$; in the Sentence 2 analysis, $88 \mathrm{msec}$. Table 3 displays the Sentence $1 /$ Sentence 2 data as a function of first and second sentence dominance.

The above results, and the pattern of RT displayed in Table 3, supported the conclusion that high-dominant second sentences were significantly primed regardless of the dominance of the priming sentence. Low-dominant sentences, on the other hand, were apparently unaffected by a preceding related sentence. A closer inspection of the tabled RT data appeared to suggest moderate priming $(31 \mathrm{msec})$ of a low-dominant Sentence 2 which followed a high-dominant priming sentence; that is, if a low-dominant second sentence cannot be primed, then the two low-dominant means at Sentence 2 should not differ. A specific test of these two points, however, failed to reach significance $(t<1.0)$. A similar test of the Sentence 2 data under high-high vs. low-high was also nonsignificant $(t<1.0)$. Neither test approached significance with a sign test.

While there was, as predicted, a significant priming effect due to semantically related stimuli, the effects of priming in conjunction with property dominance were somewhat different than was expected. The results of the study did not conform to either set of predictions in any straightforward manner. The simpler set of predictions, involving the diverse spread of priming from properties ("fanning"), was clearly not supported, since the interaction of priming (Sentence 1 vs. 2 ) and property dominance (Sentence 2 dominance) was significant.

Neither was the more detailed set of predictions supported in all aspects. The major discrepancies here were that (1) within the present procedures, a lowdominant sentence was not significantly primed even by a high-dominant primer and (2) a high-dominant second sentence was primed equally, regardless of Sentence 1 dominance. The second result is not contradictory per se, given that a specific prediction concerning priming was not possible in that condition. The first result, however, is contradictory, given the prediction
Table 3

Means (in Milliseconds), Standard Deviations, and Error Rates by Treatment Groups for Experiment I

\begin{tabular}{lcccc} 
& \multicolumn{4}{c}{ High-Dominant Sentence 1 } \\
\cline { 2 - 5 } & HD & HD & HD & LD \\
& Sentence 1 & Sentence 2 & Sentence 1 & Sentence 2 \\
\hline Mean & 1287 & 1200 & 1288 & 1280 \\
SD & 93 & 88 & 81 & 86 \\
Error Percent & 4.1 & 1.7 & 4.1 & 1.7 \\
\hline \multicolumn{4}{c}{} & \multicolumn{4}{c}{ Low-Dominant Sentence 1 } \\
\cline { 2 - 5 } & LD & HD & LD & LD \\
& Sentence 1 & Sentence 2 & Sentence 1 & Sentence 2 \\
\cline { 3 - 5 } & 1324 & 1225 & 1340 & 1311 \\
Mean & 124 & 133 & 146 & 152 \\
SD & 6.6 & 2.5 & 4.1 & 3.3 \\
Error Percent & 6.6 &
\end{tabular}

Note $-H D=$ high dominant, $L D=$ low dominant.

that a moderate but observable facilitation should have been obtained under the "high to low" sentence dominance condition. Since the priming of low-dominant sentences was also evaluated in Experiment II, further discussion of this discrepancy will be postponed.

In general, the results of this study supported the broad outlines of the Collins and Quillian (1972) model of semantic memory. Whereas this model readily predicts an interaction of priming and property dominance, no explanation of this priming effect seems available within the Smith et al. feature comparison model. Despite the relative success of Collins and Quillian at this general level, however, the more detailed predictions concerning differences among conditions were not so clearly supported.

\section{EXPERIMENT II}

Having demonstrated a significant reduction of RT due to semantic relatedness between sentences, it now becomes important to address another question: To what extent does priming with stimulus repetition yield a different estimate of priming effects per se? In other words, since many studies of priming have included stimulus repetition in the priming pairs of stimuli, it is conceivable that previous estimates of facilitation due to priming have been biased. If priming may be considered a continuous effect, such "repeated priming" might be expected to yield much stronger effects than would the more simple priming without repetition. Experiment II provided a direct test of this possibility by comparing facilitation on related pairs of stimuli which either did or did not include stimulus repetition, the "repeated priming" and "priming" conditions, respectively.

Given the basic mechanism of spreading activation in Collins and Quillian (1972), performance under "priming" and "repeated priming" conditions should 
differ only in degree, that is, "repeated priming" of a concept should simply result in more facilitation, not in different modes of processing. However, Collins and Quillian have described an alternative to the spreading activation process which might be involved in repeated priming pairs. In a statement verification task, Collins and Quillian (1969) observed very rapid RT to stimuli like "A canary is a canary," in which a decision based on "pattern matching," as opposed to semantic analysis, was possible. Such a pattern matching process could conceivably facilitate responding in a Stimulus 1/ Stimulus 2 paradigm with repeated priming sentence pairs. Interestingly, a relatively minor assumption can be added to the Smith, Rips, and Shoben (1974) model to account for repeated priming facilitation as well. This assumption would state that a concept's feature set can be retrieved more rapidly if it has been previously retrieved within some critical interval, thereby reducing overall RT to a repeated primed stimulus. This addition, however, would require many further modifications to also account for priming in the absence of repetition, as demonstrated in Experiment $I$.

In order to examine further the finding that lowdominant second sentences were not primed to an observable degree, Experiment II again included a manipulation of property dominance of Sentence 2 . The dominance variable was manipulated factorially with the type of priming to determine if the lack of facilitation might be influenced by the degree or type of priming from Sentence 1, i.e., priming or repeated priming.

Finally, a related aspect of priming, namely, the rate of decay, was addressed in Experiment II. Critical second sentences followed their first sentences after either one or four intervening, unrelated trials. This manipulation was intended to provide new information relevant to the priming and decay processes assumed by Collins and Quillian (1972). In their model, Collins and Quillian maintain that activation should decay over time and/or intervening activity, although no specific hypotheses regarding the shape of the decay function were offered. The most direct evidence for this hypothesis revealed a monotonic decay of activation across zero, one, and two intervening trials (Loftus, 1973). It should be noted, however, that this demonstration of decay involved stimulus repetition between the pairs of related stimuli. In other words, this decay effect may also be subject to the criticisms mentioned above-possible bias due to stimulus repetition. Consequently, the lag variable was manipulated factorially with type of priming and property dominance. The design was constructed such that any differential effects of type of priming across lag and across property dominance would be assessed.

\section{Method}

Subjects. Sixteen undergraduates, enrolled in upper level psychology courses, served as subjects. Subjects were paid $\$ 2$ for their services.

Stimuli. Additional true stimuli were constructed in the standard fashion and added to the pool used in Experiment I. The entire pool of stimuli is reproduced in Table 1. Forty true stimuli were drawn from this pool to serve as critical second sentences. These were divided into four groups, with 10 stimuli in each of the four cells of the property dominance (high vs. low) by type of priming (priming vs. repeated priming) matrix. The 40 first sentences which preceded the critical stimuli were of low property dominance. Twenty of these contained the same subject noun as appeared in the critical sentence paired with it, and 20 contained the related subject noun, thus forming "repeated priming" and "priming" pairs of sentences, respectively. An additional 40 sentence pairs were formed with true first but false second sentences; half of the false second sentences contained the same subject noun as the corresponding first sentences (repeated priming distractors), and half contained the related noun (priming distractors). Finally, 80 false sentences were added to equalize the number of true and false trials. Each subject noun appeared in four true and four false sentences, and each property appeared twice.

The 80 pairs ( 40 critical and 40 distractor) and the 80 additional false sentences were randomly ordered with the same restrictions as before. The sequence of 240 trials was divided into fourths for ease of presentation. For the lag manipulation, sentence pairs were separated by either one or four unrelated trials. In order to preserve comparability between lags, the sequence for Lag 1 was merely shifted where necessary to separate the sentences in the 80 pairs by four unrelated stimuli. The relative ordering of critical stimuli was preserved across the two levels of lag.

Apparatus. The apparatus from Experiment I was used with no modifications.

Design and procedure. Three major variables were manipulated. First, sentence pairs were related either with or without repetition (type of priming-repeated priming vs. priming); properties asserted in the second sentence were either high or low dominant; and, sentence pairs were separated by either one or four intervening, unrelated trials (Lag 1 vs. Lag 4). The type of priming and property dominance variables were both withinsubjects and between-stimuli. In order to maximize the number of observations per cell, lag was a between-subjects factor. Notice, however, that the same pairs of stimuli served in both levels of lag, i.e., lag was a within-stimuli effect.

Subjects were tested individually in sessions which lasted approximately $45 \mathrm{~min}$. Experiment I instructions and practice trials were administered, and feedback concerning errors was given at the end of each set.

\section{Results and Discussion}

Overall error rate was $3.9 \%$; error rate to critical stimuli was $2.2 \%$. Error proportions were examined according to treatment cells; proportions were approximately equivalent. Given this low rate, no analysis of error proportions was conducted.

An initial examination of the treatment means indicated that responses to critical stimuli were faster at Lag 4 than at Lag 1, certainly inconsistent with previous research (e.g., Loftus \& Loftus, 1974). Given the possibility that this reversal might be due to initial group differences (Lag was a between-subjects variable), RT to the practice stimuli was examined. On the average, subjects assigned to the $\mathrm{Lag} 4$ condition were $200 \mathrm{msec}$ faster during practice than subjects assigned to the Lag 1 condition. This group difference suggested strongly that the reversal for the lag effect was due to "unhappy randomization" (Kenny, 1975) into treatment groups, rather than to a treatment effect, since the data for practice stimuli were gathered prior to any experimental 
manipulation. Given the likelihood that this initial group difference accounted for the reversed lag effect, the dependent variable analyzed was the difference in RT between the priming first sentence and the critical second sentence (Sentence 1 RT minus Sentence $2 \mathrm{RT}$ ). In this situation, the Pair of stimuli which generates the difference score is considered a random factor, and significant $F^{\prime \prime}$ results provide evidence of generality to a new sample of stimulus pairs and subjects. This difference score technique should not eliminate the reversed lag effect if that reversal was due to a treatment effect, but should only correct for the apparent initial group difference.

The analysis of difference scores revealed two significant main effects. First, responses at Lag 4 showed less facilitation (i.e., smaller difference scores) than responses at $\mathrm{Lag} 1\left[\mathrm{~F}^{\prime \prime}(1,43)=5.18, \mathrm{p}<.05, \mathrm{MS}_{\mathrm{T}}=\right.$ $\left.901,050.3, \quad \mathrm{MS}_{\mathrm{e}(\mathrm{s})}=86,014.3, \quad \mathrm{MS}_{\mathrm{e}(\mathrm{st})}=102,916.9\right]$. The average difference between priming and critical stimuli at Lag 1 was $128 \mathrm{msec}(\mathrm{SD}=125)$, and this decreased to $53 \mathrm{msec}(\mathrm{SD}=87)$ at $\mathrm{Lag} 4$. This change can be considered a demonstration of the decay of priming across intervening trials. Second, the difference between high- and low-dominant sentences was significant $\left[\mathrm{F}^{\prime \prime}(1,45)=4.62, \mathrm{p}<.05, \mathrm{MS}_{\mathrm{T}}=2,104,745.0, \mathrm{MS}_{\mathrm{e}(\mathrm{s})}=\right.$ $\left.57,467.9, \mathrm{MS}_{\mathrm{e}(\mathrm{st})}=414,540.9\right]$. The difference between a low-dominant first sentence and a low-dominant second sentence was $33 \mathrm{msec}(\mathrm{SD}=101)$, while the low-dominant to high-dominant difference was $147 \mathrm{msec}$ $(\mathrm{SD}=96)$.

Table 4 presents the difference score data separately for priming and repeated priming groups for the following reasons. While the Type of Priming factor was not significant in the $F^{\prime \prime}$ analysis, an $F 1$ analysis suggested that responses to repeated priming stimuli were, on the average, $40 \mathrm{msec}$ faster than responses to "priming" stimuli $\left[\mathrm{F} 1(1,14)=6.91, \mathrm{p}<.025, \mathrm{MS}_{\mathrm{e}}=3,693.1\right]$. Type of Priming also interacted marginally with the Dominance factor $\left[\mathrm{F} 1(1,14)=3.95, \mathrm{p}<.10, \mathrm{MS}_{\mathrm{e}}=\right.$ $11,604.4]$. Despite the fact that this effect was of marginal significance, separate analyses were performed on the high- and low-dominant data. While the two $\mathrm{F}^{\prime}$ analyses revealed only a highly reliable effect of lag or high-dominant second sentences $\left[\mathrm{F}^{\prime \prime}(1,28)=10.87\right.$ $\mathrm{p}<.005, \quad \mathrm{MS}_{\mathrm{T}}=1,064,449.8, \quad \mathrm{MS}_{\mathrm{e}(\mathrm{s})}=66,030.4$ $\left.\mathrm{MS}_{\mathrm{e}(\mathrm{s})}=38,697.3\right]$, the effect of type of priming or low-dominant sentences was nonetheless quite strong in the corresponding $F 1$ analysis $[F 1(1,14)=10.24$ $\left.\mathrm{p}<.01, \mathrm{MS}_{\mathrm{e}}=7,105.1\right]$. No claim is being made tha1 performance under repeated priming conditions differec markedly from that under priming without repetition such a claim is not supported in the present data when Stimuli are included as a second random factor. Rather the point here is simply that the present data suggested a trend toward significance, particularly for repeated priming conditions with low-dominant sentences, which may be of theoretical significance in future research

In summary, the results of this experiment were fairly straight forward. The lag effect suggested that activation of concepts decayed across intervening stimuli, thereby affecting verification latency to related sentences. In this connection, note that RT for high. dominant second sentences returned to what might be approximated as baseline at $\mathrm{Lag} 4$, i.e., difference scores of approximately $100 \mathrm{msec}$ from low-dominant first sen. tences. Property dominance also affected performance significantly, and apparently did so independently of decay. Further, the difference scores for low-dominant primed (vs. repeated primed) sentences replicated the Experiment I result of no facilitation of a low-dominant second sentence. As mentioned above, this statement may need to be amended in the future, given the apparent facilitation of low-dominant sentences under the repeated priming condition; at present, however, this amendment cannot be justified statistically.

\section{GENERAL DISCUSSION}

The results of the present research may be summarized fairly briefly. First, normatively defined property dominance was a clearly significant factor in all three of the verification studies. That high-dominant sentences

Table 4

Means (in Milliseconds), Standard Deviations, Raw Means (Sentence 1 RT Minus Sentence 2 RT), and Error Rates for Difference Scores by Treatment Groups

\begin{tabular}{|c|c|c|c|c|c|}
\hline & & \multicolumn{2}{|c|}{ Priming } & \multicolumn{2}{|c|}{ Repeated Priming } \\
\hline & & $\mathrm{HD}$ & LD & HD & LD \\
\hline Lag 1 & $\begin{array}{l}\text { Mean Difference } \\
\text { SD } \\
\text { Raw Means } \\
\text { Error Percent }\end{array}$ & $\begin{array}{c}213 \\
112 \\
(1458-1245) \\
2.5\end{array}$ & $\begin{array}{c}5 \\
99 \\
(1405-1400) \\
5.0\end{array}$ & $\begin{array}{c}196 \\
60 \\
(1424-1228) \\
1.2\end{array}$ & $\begin{array}{c}95 \\
105 \\
(1396-1301) \\
5.0\end{array}$ \\
\hline Lag 4 & $\begin{array}{l}\text { Mean } \\
\text { SD } \\
\text { Raw Means* } \\
\text { Error Percent }\end{array}$ & $\begin{array}{c}95 \\
84 \\
(1453-1358) \\
3.7\end{array}$ & $\begin{array}{c}-34 \\
92 \\
(1393-1427) \\
6.2\end{array}$ & $\begin{array}{c}85 \\
45 \\
(1435-1350) \\
3.7\end{array}$ & $\begin{array}{c}63 \\
65 \\
(1408-1345) \\
2.5\end{array}$ \\
\hline
\end{tabular}

Note $-H D=$ high dominant, $L D=$ low dominant.

*Average Sentence 1 RT under Lag 1 was 1,415 msec, but was 1,262 msec under Lag 4. Consequently, a constant 153 msec has been added to the raw means for the Lag 4 condition to correct for the initial group difference. 
were always verified more quickly than low-dominant sentences suggests that property dominance is an important and relatively stable variable affecting verification processes in semantic memory. Further, property dominance is a variable which exhibits RT effects very similar to those classified as semantic similarity effects. As such, the present norms and results make contact with the larger literature in semantic verification which demonstrates the importance of semantic relatedness to performance (Kintsch, 1974; Rips et al., 1973). Finally, at a somewhat technical level, it appears that generation of property statements by intuitive (vs. normative) procedures may lead to misleading or uninformative results; that is, given the obtained interactions between dominance and priming, any experiment which fails to control explicitly for dominance of the predicate runs the risk of obscuring significant patterns of results.

Second, priming was a significant component of verification processes, both in the repeated priming situation which involved partial stimulus repetition and in the more stringent situation which excluded repetition. As noted earlier, a demonstration of priming, particularly without repetition, is quite damaging to the Smith, Rips, and Shoben (1974) feature comparison model, and consequently indicates that further revisions of the Smith et al. model are necessary to account for the present data. At a general level, then, the interdependence of priming and property dominance supports the Collins and Quillian (1972) model of semantic memory.

At a more detailed level, however, the results suggested a less successful match between the predictions from Collins and Quillian and the observed effects. The results of Experiment I indicated that a high-dominant sentence was primed regardless of the dominance of the priming sentence. This pattern implies that the functional source of priming is the subject noun concept in the priming sentence. Given this assumption, however, there should have been significant, although perhaps only moderate, priming of low-dominant sentences, since the same Sentence 1 subject nouns preceded the same second sentences. While the conclusion of no priming relies on the null hypothesis, the pattern was replicated in Experiment II. Consequently, it appears that activation spreads primarily from the subject noun concept, and only highly related concepts and properties are the targets of such a spread of priming. This interpretation suggests that when a sentence is presented (e.g., "A robin has eyes"), priming spreads primarily to those concepts highly related to the subject noun (e.g., from ROBIN to FEATHERS, WINGS, BIRD, SPARROW). A subsequent high-dominant related sentence ("A sparrow has wings") will thereby be verified quickly, since both concepts are likely to have been activated; whereas verification time for a subsequent low-dominant related sentence ("A sparrow has feet") will still depend on the spread of activation from an unprimed (FEET) concept. This interpretation meshes well with the Collins and Quillian search metaphors of the "slowest runner" (FEET to SPARROW) and "elapsed time until intersection threshold" (Collins \& Quillian, 1972; Collins \& Loftus, 1975).

This interpretation coincides, with one exception, with the results from Experiment II as well. Once again, a low-dominant sentence in the priming condition was apparently not facilitated by a preceding related sentence, while high-dominant sentences were. The only exception to this pattern was the observations for low-dominant sentences under the repeated priming condition. The difference scores suggested that a lowdominant sentence can be primed, but only if that priming involves repetition of the subject noun. It is not clear, from these results, whether this facilitation was due to a "true" priming of the second sentence or to a pattern match process which might be performed on the repeated subject noun. If priming was involved, one might expect a significant effect of lag on low-dominant sentences, indicating decay of this priming. The absence of such an effect, however, suggested that a pattern match or purely repetition effect was involved. While not a necessary prediction, it would be reasonable to expect that a pattern match or repetition facilitation would be additive with a priming facilitation; however, this was not the case under conditions of high-dominant critical sentences. This question cannot be resolved at present, since the difference involving types of priming for low-dominant sentences was not statistically significant when stimuli were included as a random factor.

Bearing this tentative qualification in mind, the present results supported the following conclusions: Priming, whether or not it involves repetition, spreads primarily from a subject noun concept to highly related concepts and properties; significant facilitation due to this spread of activation is observed only when both of the concepts in the subsequent critical stimulus have been primed; and, when both target concepts have been activated, the resulting facilitation affects performance in a decreasing fashion as the initial level of priming decays.

\section{NOTE}

1. The $F^{\prime \prime}$ ratio is a quasi-F formed by the addition of mean squares, as opposed to the $F^{\prime}$ ratio which can involve addition and/or subtraction; as such, $F^{\prime \prime}$ eliminates the possibility of a negative mean square in the quasi-F ratio. $F^{\prime \prime}$ is preferred here since it does not involve combinations of $F^{\prime} s$ from separate analyses, as does the min $F^{\prime}$. One difficulty, however, concerns appropriate indices of variability to report along with $\mathrm{F}^{\prime \prime}$. That is, since both the MS numerator and MS denominator are combinations, neither is a straightforward mean square for treatment or error. Accordingly, all $\mathrm{F}^{\prime \prime}$ statements here will include MS treatment $\left(\mathrm{MS}_{\mathrm{T}}\right)$, MS error for subjects $\left(\mathrm{MS}_{\mathrm{e}}(\mathrm{s})\right.$ ), and $M S$ error for stimuli $\left(\mathrm{MS}_{\mathrm{e}(\mathrm{st})}\right)$ from the $\mathrm{F}^{\prime \prime}$ analysis, where:

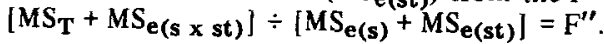




\section{REFERENCES}

ANDERson, J. R. Retrieval of propositional information from long-term memory. Cognitive Psychology, 1974, 6, 451-474.

Battig. W. F., \& Montague, W. E. Category norms for verbal items in 56 categories: A replication and extension of the Connecticut category norms. Joumal of Experimental Psychology Monograph, 1969, 80(3, Pt. 2).

Clark, H. H. The language-as-fixed-effect fallacy: A critique of language statistics in psychological research. Journal of Verbal Learning and Verbal Behavior, 1973, 12, 335-359.

Collins, A. M., \& LofTus, E. F. A spreading activation theory of semantic processing. Psychological Review, 1975, 82, 407-428.

Collins, A. M., \& Quillian, M. R. Retrieval time from semantic memory. Jourmal of Verbal Learning and Verbal Behavior, 1969, 8, 240-248.

Collins, A. M., \& Quillinn, M. R. Facilitating retrieval from semantic memory: The effect of repeating part of an inference. Acta Psychologica, 1970, 33, 304-314.

Collins, A. M., \& Quillian, M. R. How to make a language user. In E. Tulving \& W. Donaldson (Eds.) Organization of memory. New York: Academic Press, 1972.

Conrad, C. Cognitive economy in semantic memory. Journal of Experimental Psychology, 1972, 92, 149-154.

Forbach, G. B., Stanners, R. F., \& Hochhaus, C. Repetition and practice effects in a lexical decision task. Memory \& Cognition, 1974, 2, 337-339.

Glass, A. L., Holyoak, K. J., \& O'Dell, C. Production frequency and the verification of quantified statements. Journal of Verbal Learning and Verbal Behavior, 1974, 13, 237.254.

Haviland, S. E., \& Clark, H. H. What's new? Acquiring new information as a process in comprehension. Journal of Verbal Learning and Verbal Behavior, 1974, 13, 512-521.

KENNY, D. A. A Quasi-experimental approach to assessing treatment effects in the nonequivalent control group design. Psychological Bulletin, 1975, 82, 345-362.
KINTSCH, W. The representation of meaning in memory Hillsdale, N.J: Lawrence Erlbaum Associates, 1974.

KuČERA, H., \& Francis, W. N. Computational analysis o present-day American English. Providence: Brown Universit Press, 1967.

Lofrus, E. F. Activation of semantic memory. Americal Journal of Psychology, 1973, 86, 331-337.

LofTus, E. F. Spreading activation within semantic categories Comments on Rosch's "Cognitive representations o semantic categories." Journal of Experimental Psychology General, 1975, 104, 234-240.

Loftus, G. R., \& Loftus, E. F. The influence of one memor. retrieval on a subsequent memory retrieval. Memory \& Cognition, 1974, 2, 467-471.

Meyer, D. E., \& SchVANeveldt, R. W. Facilitation is recognizing pairs of words: Evidence of a dependence between retrieval operations. Joumal of Experimenta Psychology, 1971, 90, 227-234.

Rips, L. J., Shoben, B. J., \& Smith, E. E. Semantic distancı and the verification of semantic relations. Journal $o$ Verbal Learning and Verbal Behavior, 1973, 12, 1-20.

Rosch. E. Cognitive representations of semantic categories Journal of Experimental Psychology: General, 1975, 104 192-233.

Smith, E. E., Rips, L. J., \& Shoben, E. J. Semantic memor and psychological semantics. In G. H. Bower (Ed.), Thi psychology of learning and motivation (Vol. 8). New York Academic Press, 1974.

Smith, E. E., Shoben, E. J., \& Rips, L. J. Structure anc process in semantic memory: A feature model for semanti decisions. Psychological Review, 1974, 81, 214-241.

WINER, B. J. Statistical principles in experimental design New York: McGraw-Hill, 1971.

(Received for publication November 10, 1975; revision accepted January $7,1976$. ) 